\title{
El proyecto de investigación social como instrumento integrador de la praxis para los futuros/as trabajadores/as sociales
}

\author{
The social research project as an integrating instrument of praxis for future \\ social workers
}

\author{
$\mathrm{M}^{\mathrm{a}}$ de las Mercedes BotiJA YAGÜE \\ Universidad de Valencia \\ mercebotija@gmail.com \\ José Javier NAVARRO PÉREZ \\ Universidad de Valencia \\ j.javier.navarro@uv.es
}

Recibido: 06/06/2013

Revisado: $28 / 06 / 2013$

Aceptado: 23/02/2015

Disponible on line: 10/06/2015

\begin{abstract}
Resumen
El presente trabajo muestra los resultados de una investigación-intervención aplicada a los alumnos de cuarto curso de Grado en Trabajo Social. En este documento se articula una metodología de enseñanza colaborativa, significativa y crítica a partir de las evidencias de la práctica del Trabajo Social. Como conclusiones de este estudio se justifica el uso de la metodología Blended Learning en el espacio universitario. Se evidencia como con la participación y cooperación colectiva se favorece el desarrollo de competencias investigadoras, y se señala el proyecto de investigación social como herramienta que permite vincular las prácticas externas del alumnado con los elementos teóricos de la disciplina.

Palabras clave: Proyecto de investigación social, práctica basada en la evidencia, aprendizaje significativo, trabajo cooperativo, competencias, investigación.
\end{abstract}

\begin{abstract}
This paper presents the results of a research-intervention applied to fourth-year students of Degree in Social Work. The study implements a collaborative, significant and critical teaching methodology considering evidence-based practice of social work. The conclusions show how the use of the blended learning methodology in the higher education is justified. Furthermore is clear how the participation and the collective cooperation foster the development of research competences. Likewise it is pointed out how the social research project is a tool to link the external practices of students with the theoretical elements of the discipline.

Keywords: Social Research Project, evidence-based practice, significant learning, cooperative work, competitions, investigation.

Referencia normalizada: Botija Yagüe, Ma M., y Navarro Pérez, J. J. (2015): «El proyecto de la investigación social como instrumento integrador de la praxis para los futuros/as trabajadores/as sociales». Cuadernos de Trabajo Social, 28(1): 49-60.

Sumario: Introducción: La práctica basada en la evidencia científica en el Espacio Europeo de Educación Superior. 1. Marco teórico: educación crítica en el aprendizaje significativo y la cooperación por medio de Blended Learning.

2. Metodología. 3. Resultados. 4. Discusión de resultados. 5. Conclusiones. 6. Referencias bibliográficas.
\end{abstract}

Introducción: La práctica basada en la evidencia científica en el Espacio Europeo de Educación Superior

La denominada Declaración de Bolonia ha generado un proceso de cambio en las universida- des europeas y una interesante proliferación por introducir, en el corpus académico del Espacio Europeo de Educación Superior, la denominada práctica basada en la evidencia definida como «la integración de la mejor evidencia extraída 
de los estudios de investigación con la pericia profesional y los valores del usuario» (Sackett, Strauss, Richardson, Rosenberg y Haynes, 2000), que tiene su origen en Medicina y que se resume en el método para tomar decisiones que formula Mooney (2004):

1. Formulación de una pregunta clínica clara y precisa a partir de un problema clínico dado.

2. Búsqueda de la literatura de artículos originales relevantes y apropiados para el problema.

3. Evaluación crítica de la validez y utilidad de los artículos encontrados

4. Aplicación de los resultados a la práctica clínica del paciente tomando en cuenta su contexto y sus preferencias.

Una de las consecuencias principales de la proliferación de la práctica basada en evidencias es su contagio a otras disciplinas y en concreto a Trabajo Social, como ya atestigua Morago (2009), cuando analiza el acercamiento de la Universidad a los Servicios Sociales con la finalidad de aproximar la investigación y la docencia a la práctica profesional. Esto supone la aceptación de un nuevo modelo de enseñanza-aprendizaje que, en el caso del sistema internacional de créditos, permite la adquisición de las competencias que se presentan en el Libro Blanco del Trabajo Social, coherentes con la práctica basada en la evidencia. Es decir, se ha de crear un patrón didáctico capacitado para el abordaje transversal, tanto de competencias generales como de las específicas, y apto para alcanzar los resultados derivados del aprendizaje reflejados en el título de Grado en Trabajo Social verificado por la ANECA en la línea de incluir competencias relativas a la capacidad para transmitir y potenciar la igualdad de oportunidades; trabajar y valorar de manera conjunta a individuos, familias y comunidades; capacidad para planificar, revisar y evaluar; trabajar en redes multidisciplinares; investigar, analizar y evaluar las mejores prácticas del Trabajo Social, etc.

Este nuevo modelo de enseñanza, basado en el Sistema Europeo de Transferencia de Créditos, cuantifica tanto el trabajo realizado por el alumno de manera no presencial como el seguimiento de las horas de clase teóricas presenciales. De esta manera, acorde con el Trabajo Social, se fomenta en el alumno la emancipación hacia su futuro profesional. Acorde con Medina,
Domínguez y Sánchez (2013) el aprendizaje basado en competencias implica evolucionar desde un planteamiento centrado en el profesor y en la exposición de clases magistrales, a otro orientado al estudiante y a sus potencialidades para articular de manera autónoma los contenidos que requiere la práctica real. De este modo, la formación por competencias coordina los planteamientos didácticos con la enseñanza, el aprendizaje y la evaluación, ubicando al estudiante en el centro del proceso para orientarle a la obtención de sus objetivos, en el modo de resultados, que le permitan desarrollar competente y eficazmente su trabajo (Aguado y Arranz, 2005). Esto «permite incorporar al alumno en el marco académico que legitima la disciplina» (Campanini, 2009, p. 13).

Un programa docente que incorpora la evidencia científica y fomenta el desarrollo de la formación en competencias investigadoras en los nuevos grados requiere un cambio metodológico más extensivo, si deseamos ahondar en aprendizajes significativos que sean útiles a los futuros profesionales. El abordaje de estos nuevos itinerarios de formación, precisan escenarios innovadores $\mathrm{y}$, en consecuencia, poner la tecnología al servicio del aprendizaje cooperativo, considerando como referencia la adquisición de competencias para trabajar en grupo (Gil, Rico y Sánchez-Manzanares, 2008). Este trabajo grupal supone un elemento esencial para los futuros profesionales del Trabajo Social, cuya labor probablemente se desarrolle en equipos interdisciplinares, y apoyarlo en investigaciones de alta calidad presupone construir, desde la evidencia, una sólida práctica.

Desde la perspectiva anteriormente descrita, y atendiendo a los cambios que para sí reclama el Espacio Europeo de Educación Superior relativos a los diferentes estudios universitarios, el Departamento de Trabajo Social de la Universidad de Valencia optó por impartir a los estudiantes de la primera promoción de $4^{\circ}$ de Grado en Trabajo Social, por medio de la metodología conocida como Blended Learning, un taller especializado en la realización de proyectos de investigación para el Trabajo Social. El objetivo de dicho taller era incentivar la adquisición de competencias investigadoras en los futuros profesionales de Trabajo Social desde la práctica basada en la evidencia. En consecuencia se trata de establecer una relación directa con la prác- 
tica del Trabajo Social, capaz de configurar en un mismo nivel teoría y práctica. La articulación de esta relación derivará en el resultado final que posteriormente se analizará tras enmarcar este artículo desde el marco teórico

\section{Marco teórico: Educación crítica en el aprendizaje significativo y la cooperación por medio de Blended Learning}

Tomando como referencia a Paulo Freire (1979) que propone un modelo de intervención educativa que fomente el análisis crítico de la realidad, el aula ha de convertirse en un escenario de reflexión, para apoyar la teoría de aprendizaje significativo de Ausubel (2000) y la teoría del aprendizaje cooperativo de Johnson y Johnson (1990), construcciones teóricas que dan soporte a la metodología Blended Learning.

En el aprendizaje significativo se destaca la experiencia previa con la que el alumnado mostrará una predisposición más elevada para relacionar el nuevo conocimiento con el ya adquirido (Ausubel, 2000). Por ello, los ejemplos con los que se trabajó durante todo el proceso estaban próximos a la realidad cotidiana de los estudiantes, derivada de sus experiencias, utilizando para ello las prácticas profesionales que se encontraban realizando en ese momento. Las prácticas externas supusieron el puerto en el que encallar los aportes teóricos sobre los que desarrollar los futuros proyectos de investigación y en los que sustentar posteriormente las intervenciones.

En la docencia la definición del trabajo cooperativo incluye múltiples perspectivas; pero en esencia sustituye la optimización de las acciones productivas y competitivas por otro tipo de prácticas organizativas «basadas en el trabajo en equipo y en el alto desempeño» (Johnson, Johnson y Holubec, 1999, p. 4). En la experiencia que se describe en este artículo se optó por un trabajo cooperativo, teniendo como base múltiples estudios que atestiguan sus virtudes (Susman, 1998; Slanvin y Cooper, 1999; Coll, Maurí y Honrubia, 2006; Garaigordobil, 2007 y 2012; Aronson y Patnoe, 2011). Este plano de colaboración transciende el aula y a los alumnos, y fomenta también la colaboración de los docentes de la titulación y de los trabajadores sociales en activo que, a través de la participación colaborativa, podrían complementar y optimizar el modelo de aprendizaje que presenta- mos. Esto supone incorporar un sistema de transferencia de conocimiento guiado capaz de proporcionar oportunidades para la ejecución y el manejo de las habilidades que posteriormente regirá el trabajo desde el plano colaborativo

La articulación de la reflexión crítica, el aprendizaje significativo y el trabajo cooperativo, como base para formar a profesionales capacitados en el denominada práctica basada en la evidencia, se realizó mediante una metodología dual que articula diferentes escenarios de participación, presenciales y no presenciales por medio de las nuevas tecnologías. Es lo que se ha optado por denominar metodología Blended Learning, defina por Silva (2011) como un aprendizaje dual capaz de articular el espacio físico presencial con el virtual. Esta metodología, nacida de las limitaciones del denominado e-learning - enseñanza exclusivamente virtual- que no es capaz de prestar la atención suficiente a ciertas variables educativas y didácticas (Cabero y Llorente, 2005), y de la masificación de las clases presenciales, ha sido considerada como una de las innovaciones más fructíferas para el aprendizaje (Aiello, 2004). El Blended Learning, como metodología semipresencial, permite unificar las ventajas de otro tipo de metodologías. En esencia se trata de desarrollar un instrumento flexible para el desempeño, acompañado de soporte e-learning con acceso al colectivo total de alumnos y capaz de conjugarse con el modelo de aprendizaje en el que nos apoyamos.

Coherente con las ventajas de esta metodología didáctica, se posibilita aunar sinergias y en la que compatibilizamos diferentes opciones: elearning y aprendizaje individual, estableciendo un ritmo personalizado para cada estudiante; tutorías individuales y tutorías grupales; taller presencial y trabajo cooperativo a través de la instrumentalización de la técnica puzzle, en la que son los propios alumnos, con apoyo del profesor, los que lideran el aprendizaje propio y el de sus compañeros, retroalimentándose en conocimientos, provocando una interdependencia positiva al trabajar juntos, de modo que los objetivos de los participantes se hallen vinculados con el objetivo común, en este caso el diseño de proyectos de investigación social. Desde esta perspectiva, el proyecto de investigación social supone el punto de partida que guía los diagnósticos fiables de la realidad y, en consecuencia, 


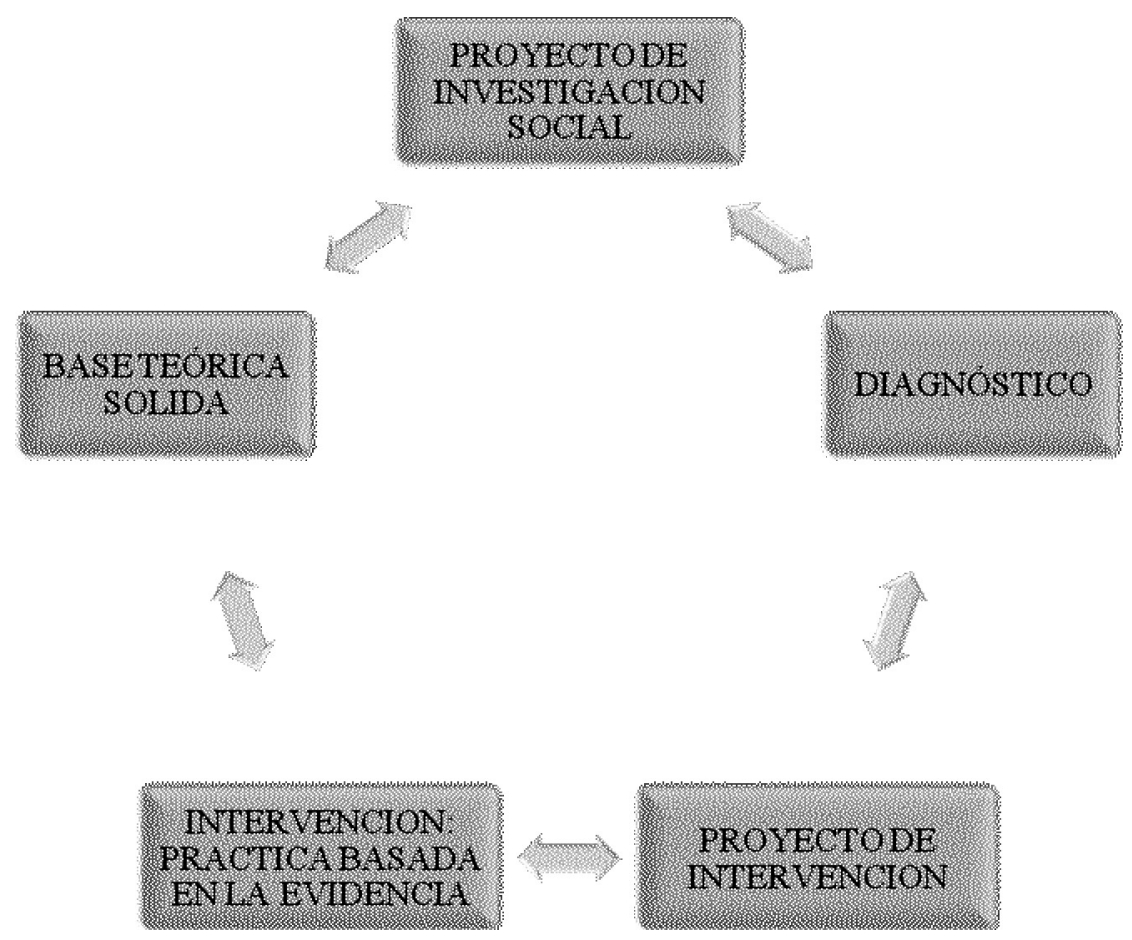

Figura 1. Proceso de la práctica basada en la evidencia en Trabajo Social.

las intervenciones, que se convertirían en las prácticas basadas en la evidencia.

\section{Metodología}

En conexión con el objetivo general y los factores específicos anteriormente citados, se utilizó una metodología cuantitativo-cualitativa. En primer lugar, sobre una población total de 183 estudiantes universitarios de Trabajo Social se obtuvo una muestra de 78 alumnos de último curso de Grado a los que se pasó cuestionarios con preguntas tanto cerradas como abiertas con el fin de observar el interés por la investigación así como en relación a la adquisición de competencias analíticas. Con ello obtuvimos un marco referencial sobre las necesidades investigadoras en el ámbito de la formación académica. En segundo lugar, se diseñó un material ad hoc en relación al diseño de proyectos de investigación al que los alumnos pudieron tener acceso por medio de la plataforma virtual.

El tercer paso consistió en la realización del taller «Diseño de Proyectos de Investigación Social», con una metodología de aprendizaje colaborativo en sesión presencial en la que, por medio de la técnica puzzle y con un caso práctico, se elaboró un proyecto de investigación, utili- zando técnicas grupales, favoreciendo la participación colectiva de todo el alumnado presente en el taller. Para la implementación de esta técnica el grupo se dividió en tríos, formando equipos. A cada uno de ellos se le encomendó el diseño de una parte del proyecto de investigación, de manera que la realización de la totalidad del documento estaba determinada por la colaboración mutua, la puesta en común y el debate por el resto de compañeros. El proyecto de investigación grupal se trasladó al aula virtual para que la totalidad de los estudiantes tuvieran acceso inmediato a este material elaborado colectivamente. Este mismo material también cumplió la función de poder ser evaluado en referencia a la adquisición de competencias.

Tras la realización del taller, en cuarto lugar, se pasó un test con el propósito de comparar la adquisición de competencias en materia investigadora, utilizando la técnica test-retest que permitía comparar el antes y después de la realización del taller. Así mismo se realizó un cuestionario de satisfacción en el que se abordaba la metodología aplicada y se incluían diferentes apartados en los que el alumnado podría evaluar su experiencia de aprendizaje, estableciendo además, propuestas para mejorar la 
experiencia en los próximos cursos académicos.

Finalmente, los alumnos diseñaron su propio proyecto de investigación, por medio de tutorías presenciales y virtuales. Estos trabajos se compararon y evaluaron con los proyectos de investigación realizados por los estudiantes de los cursos académicos anteriores de nivelación o adaptación al Grado ${ }^{1}$, en los que no se habían desarrollado ni el taller de proyectos de investigación social ni las actividades asociadas a él, con la finalidad de comparar la eficiencia de esta experiencia además de valorar las reacciones derivadas de la experiencia colectiva.

Este marco metodológico facilitó la evaluación de la adquisición de competencias investigadoras por los alumnos así como la valoración de la utilización de la metodología Blended Learning en el espacio universitario, ya que esta experiencia combina los elementos más efectivos de la clase en grupo presencial y de las nuevas tecnologías. Para ello, como muestra el Cuadro 1, se

\begin{tabular}{|c|c|}
\hline Objetivos & Actividades \\
\hline $\begin{array}{l}\text { Etapa } 1 \\
\text { — Detectar necesidades formativas e investigado- } \\
\text { ras del alumnado de último año de grado. }\end{array}$ & $\begin{array}{l}\text { - Diseño de cuestionario. } \\
\text { - Pasar cuestionario al alumnado. } \\
\text { - Test para evidenciar competencias reales del } \\
\text { alumnado. }\end{array}$ \\
\hline $\begin{array}{l}\text { Etapa } 2 \\
\text { - Diseñar desde la perspectiva Blenden Learning } \\
\text { una metodología de enseñanza significativa y co- } \\
\text { operativa. } \\
\text { - Desarrollar en el alumnado universitario com- } \\
\text { petencias teórico prácticas en relación a la investi- } \\
\text { gación. }\end{array}$ & $\begin{array}{l}\text { - Elaboración de material específico para la rea- } \\
\text { lización de proyectos de investigación. } \\
\text { - Trasladar este material al aula virtual. } \\
\text { — Exponer en clase presencial el taller: diseño de } \\
\text { proyectos de investigación. }\end{array}$ \\
\hline $\begin{array}{l}\text { Etapa } 3 \\
\text { - Fomentar habilidades cooperativas útiles en su } \\
\text { inserción laboral. } \\
\text { - Potenciar la capacidad reflexiva y crítica del } \\
\text { alumnado. }\end{array}$ & $\begin{array}{l}\text { - Cuestionario satisfacción del taller. } \\
\text { - Trasladar a la plataforma virtual el proyecto de } \\
\text { investigación común realizado por los alumnos. } \\
\text { - Observación de la dinámica del taller, focali- } \\
\text { zando la atención en las actividades que requieren } \\
\text { consensos y acuerdos para alcanzar compromisos } \\
\text { comunes en el desempeño del Trabajo Social. }\end{array}$ \\
\hline $\begin{array}{l}\text { Etapa } 4 \\
\text { - Analizar las competencias adquiridas por el } \\
\text { alumnado. } \\
\text { - Explorar el grado de satisfacción de los alum- } \\
\text { nos en la utilización de esta metodología. } \\
\text { - Evaluar competencias investigadoras adquiri- } \\
\text { das a nivel individual. }\end{array}$ & $\begin{array}{l}\text { - Test y retest. } \\
\text { - Evaluación del proyecto de investigación so- } \\
\text { cial realizado en común. } \\
\text { - Diseño de proyectos de investigación de mane- } \\
\text { ra individual vinculados con las practicas externas }\end{array}$ \\
\hline $\begin{array}{l}\text { Etapa } 5 \\
\text { - Evaluar metodología Blended Learning aplica- } \\
\text { da tanto al Diseño de Proyectos de Intervención } \\
\text { como de Investigación Social. }\end{array}$ & $\begin{array}{l}\text { - Tutorías presenciales y virtuales. } \\
\text { - Pase al alumnado de cuestionario en referencia } \\
\text { a la experiencia metodológica. }\end{array}$ \\
\hline
\end{tabular}

Cuadro 1. Etapas del proyecto.

${ }^{1}$ Alumnos procedentes de las antiguas diplomaturas de Trabajo Social que se habían matriculado en el Curso de adaptación o nivelación durante los cursos académicos 2010/11 y 2011/12, para adecuar sus estudios a los actuales grados universitarios. 
describen los objetivos del proyecto atendiendo tanto las diferentes etapas como a las actividades asociadas a cada una de ellas.

\section{Resultados}

Para la descripción de los resultados y afín a los diferentes momentos de la investigación, mostramos los hallazgos derivados tanto del cuestionario inicial realizado con los alumnos, como la evaluación de las competencias adquiridas en el taller, el cuestionario de satisfacción en torno al taller de aprendizaje colaborativo, la metodología Blended Learning y los proyectos de investigación social, ya sea el realizado de forma grupal como los realizados de forma individual.

En el marco de la primera etapa, el cuestionario inicial, se detectó que una mayoría absoluta mostraron interés por la investigación (más del 90 por ciento) y en consecuencia consideraron relevante la incorporación al plan de estudios de asignaturas en esta materia ( 90 por ciento) porque absolutamente todos refirieron que la investigación incidía de manera directa en la intervención con individuos, grupos y comunidades; es decir, en las buenas prácticas que se realizan en el espacio profesional cotidiano.
En lo que se refiere a la percepción que los propios alumnos tenían sobre sus competencias investigadoras, estos mostraron tener dificultades en la utilización adecuada de las diferentes metodologías aplicadas y en la proyección práctica derivada de estas (obsérvese la Figura 2).

En relación a las competencias prácticas (realizar proyecto de investigación social, hacer diagnóstico o relacionar teoría y práctica), los alumnos se mostraban inseguros y con marcadas limitaciones.

En lo que se refiere a las competencias metodológicas de investigación (conocer y utilizar técnicas cuantitativas, cualitativas, de diseño, identificar bases de datos, búsquedas bibliográficas, etc.) es en el apartado donde mostraron más seguridad.

Por último, en relación a las competencias para transmitir los resultados de las investigaciones (utilización normas de estilo $\mathrm{APA}^{2}$, redacción eficaz y científica) se volvieron a evidenciar ciertas dudas. Estas limitaciones, que ya se habían observado en la percepción del alumnado, quedaron patentes nuevamente tras la realización el test, donde el 50 por ciento del alumnado presentó dificultades para integrar las competencias investigadoras

Incidencia de la investigación en las buenas prácticas

Incorporación de la investigación

Interés por la investigación

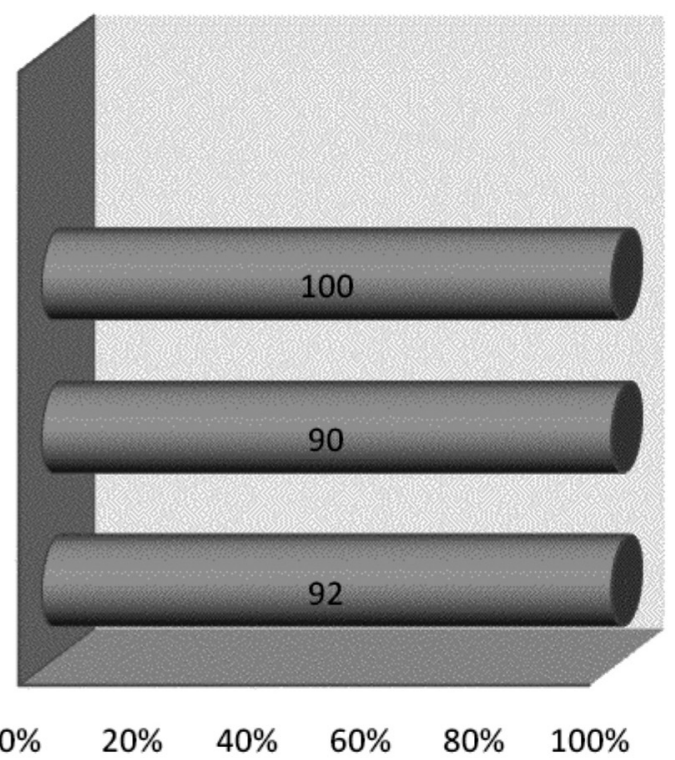

Figura 2. Percepción del alumnado sobre la investigación.

${ }^{2}$ El denominado estilo APA es el estándar adoptado por la Asociación Estadounidense de Psicología (American Psychological Association, APA) y que gran parte de las áreas de humanidades y ciencias sociales utiliza en la citación de sus textos científicos. Además estos estándares contienen diferentes directrices para los aspectos relacionados con la redacción. 
En lo que se refiere al segundo momento, entre los materiales específicos que se realizaron (bibliografía sobre proyectos de investigación social, reglas APA, redacción eficaz, etc.) destacó el interés que mostraron los alumnos por la plantilla de proyecto de investigación social (véase la Figura 3), ya que ello les permitió configurar mental y físicamente la realidad que requerían para la elaboración del diseño.

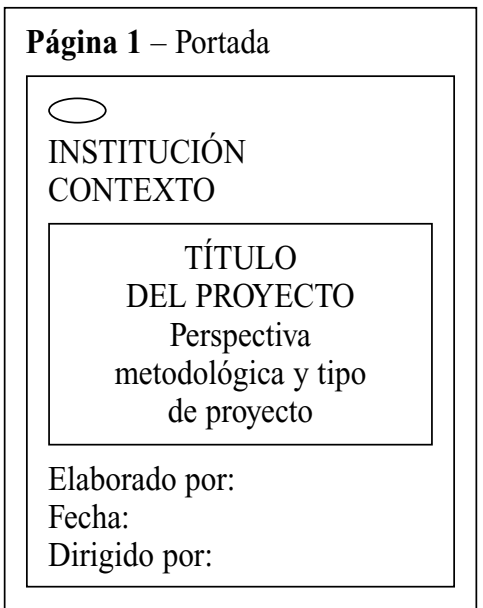

Páginas 4/5 - Formulación del problema/construcción del objeto de investigación

- Tema

- Ámbito temático

- Ámbito poblacional

- Localización

— Formulación de hipótesis

\section{Página 2 - Índice}

I. Justificación

II. Formulación del objeto

III. Objetivos

IV. Metodología

V. Cronograma

VI. Recursos

VII. Bibliografía

VIII. Anexos

- Objetivo/s general

— Objetivos específicos

Página 6 - Objetivos
Página 3 - Justificación

- Antecedentes

- Delimitación del campo

- Relevancia social

- Beneficios del proyecto

- Marco teórico, legislativo y definición conceptual

Página 7 - Metodología y técnicas de la investigación

- Técnicas

— Variables

Página 9 - Recursos

\begin{tabular}{|l|l|l|l|l|l|l|l|l|l|}
\hline Obj. & Act/tareas & En. & Fb. & Mr. & Ab. & My. & Jn. & .. & Responsable \\
\hline \multirow{2}{*}{$\sim$} & & & & & & & & & \\
\cline { 2 - 9 } & & & & & & & & & \\
\hline & & & & & & & & & \\
\hline \multirow{2}{*}{$\therefore$} \\
\cline { 2 - 9 } \\
\cline { 2 - 9 }
\end{tabular}

Página 10 - Bibliografía

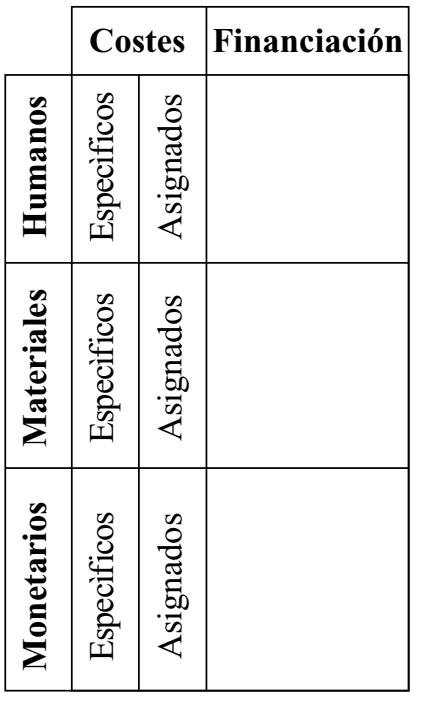

Figura 3. Plantilla proyecto de investigación. 
En lo que se refiere a la ejecución del taller «Diseño de Proyectos de Investigación» Etapa 3 , mediante la clase presencial y la metodología colaborativa, se pudo observar como resultado que el total del alumnado había participado activamente de la dinámica de la clase mostrando en diferentes niveles capacidades colaborativas, reflexivas y críticas. El mismo porcentaje se encontraba entre satisfecho y muy satisfecho con los objetivos de la actividad y más del 90 por ciento mostró satisfacción total con los contenidos del taller.

En relación a la metodología colaborativa, el 86 por ciento de los estudiantes refirió que la relevancia de la metodología colaborativa era un factor sustancial que favorecía el desarrollo de las capacidades de afrontamiento de los problemas cotidianos, a los que se enfrentan los encuestados durante su periodo de prácticas, y era imprescindible esta metodología para dar respuesta a los problemas relacionados con el espacio profesional, ya que exige implicación y compromiso mutuo para responder a las situaciones complejas. Además las tres cuartas partes de los encuestados valoraron la metodología de aprendizaje colaborativo en el espacio universitario como de «elevado interés» para instrumentalizar, de alguna manera, las dinámicas representativas de las organizaciones y diversificar las funciones en los equipos de trabajo. Superior a este mismo porcentaje (el 81 por ciento) expuso que las técnicas cooperativas favorecían también un mayor dinamismo en las aulas y posibilitaban la creatividad y el encuentro de intereses con otros estudiantes participantes.

Como aportaciones para mejorar el taller presencial, se hacen necesarias más actividades similares a la descrita en este paper, con profesionales de la práctica, y más tiempo de dedicación, por considerarse que este tipo de dinámicas vinculan de manera positiva la teoría adquirida con la práctica profesional del Trabajo Social.

Después de desarrollar el taller se realizó un test en el que se evaluaron tanto las competencias adquiridas como el proyecto de investigación grupal realizado. Los resultados que se desprenden, de la que hemos optado por denominar etapa cuatro, por medio del test-retest arrojan una observación: una importante mayoría demostró haber adquirido competencias investigadoras. Tanto en lo que se refiere a las competencias prácticas (94 por ciento), como competencias metodológicas (95 por ciento) y en competencias para transmitir los resultados de las investigaciones (91 por ciento).

En referencia al proyecto de investigación social realizado de manera colaborativa, se corroboró la adquisición de las competencias en las diferentes partes del proyecto. Para ello se evaluó de 0 a 10 cada apartado, comprobándose una estimación muy positiva. Existe el riesgo de caer en alusiones subjetivas cuando se trata de las percepciones individuales. Coincidimos con Pastor (2011) en que las percepciones y las expectativas de los sujetos pueden generar resultados confusos; sin embargo, el nivel de adquisición de competencias, de participación, responsabilidad y logro en la tarea descartó cualquier duda inicial. Así se evidencian los hallazgos en la Figura 4.

En referencia a la etapa quinta, los resultados obtenidos en el diseño de proyectos de investigación social de manera individual y la evaluación de la metodología Blended Learning por el alumnado, hallamos lo siguiente: en primer lugar los proyectos diseñados por los alumnos incrementaron en calidad respecto a los años anteriores, en los que no se había programado ni el taller presencial de proyectos de investigación ni realizado el aprendizaje por medio de la metodología Blended Learning. En segundo lugar los alumnos expresaron, entre sus conclusiones, que este tipo de metodologías, directamente vinculadas con la práctica, podían favorecer relevantes contribuciones para su aprendizaje, pero también para la sociedad al aportar investigaciones empíricas extraídas de la realidad social. Como tercer aspecto a destacar, en el marco de la elaboración de diagnósticos sociales a partir del desarrollo de metodologías colaborativo-participativas, donde habían prevalecido la discusión y la reflexión respecto a los datos obtenidos, los estudiantes valoraron de sumo interés esta óptica, y a que el 86 por ciento señaló como «bastante importante» y «muy importante» el hecho de poder identificar, organizar y usar la información particular de los usuarios de manera ética para construir diagnósticos fiables y eficaces que contribuyesen al diseño de proyectos de investigación social de calidad, amparados en criterios científicos. Cabe señalar, en cuarto lugar, que una proporción superior al 90 por ciento de los estudiantes participantes señalaron como «muy interesante» la im- 

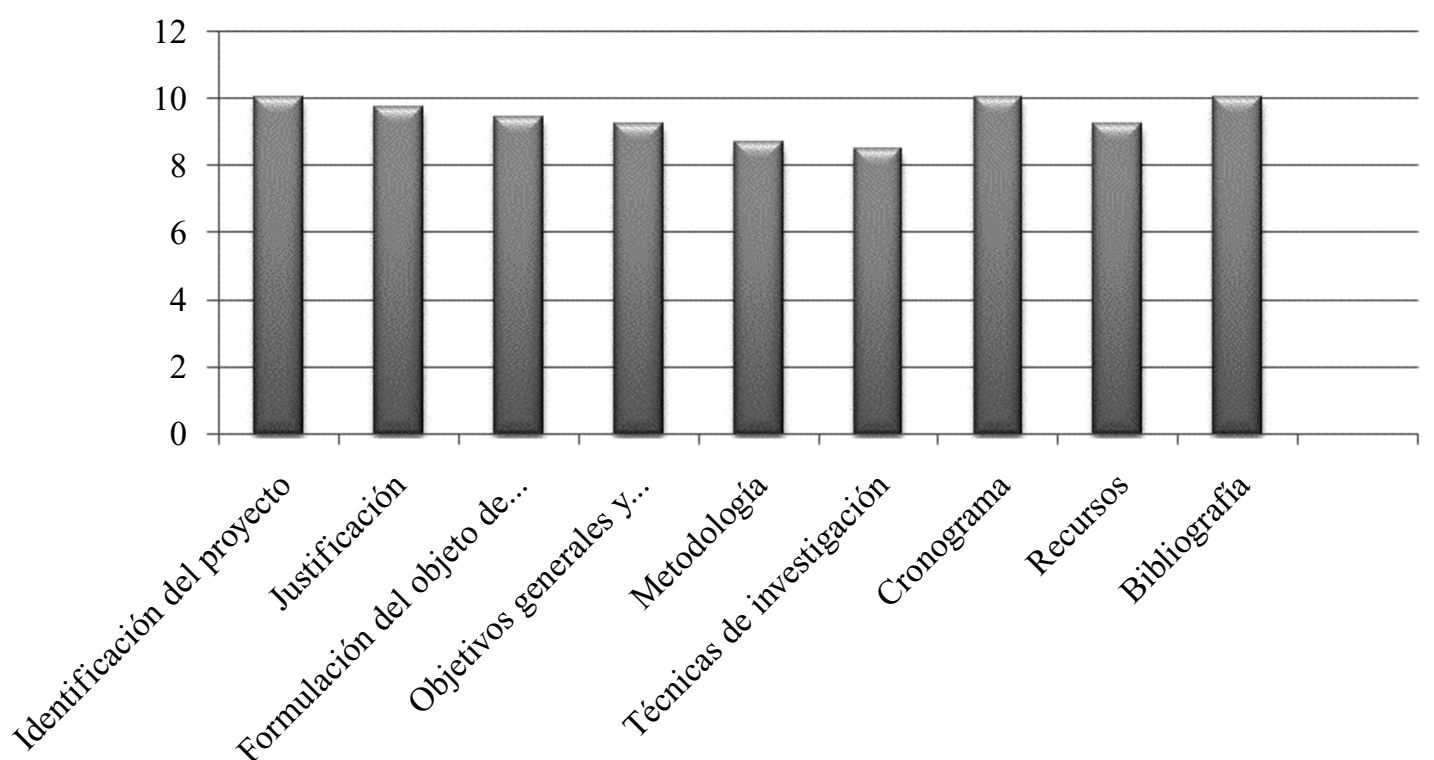

Figura 4. Adquisición de competencias en las diferentes partes del proyecto de investigación valoradas de 0 a 10.

portancia de poder relacionar teoría y práctica del Trabajo Social a través de este tipo de acciones.

Con referencia al uso de la Blended Learnimg, se especifica que el total de estudiantes encuestados desconocía esta metodología y que al terminar el taller el 68 por ciento se interesó por su estructura y su dinamismo para poder trasladarla a otros contextos de la acción social (Practicum) e incluso para poder flexibilizar su uso mediante la inclusión de las herramientas virtuales ya existentes (Dropbox, Google.docs, etc.). Se añade que, en el plano personal, una proporción del alumnado superior al 65 por ciento expresó que la actividad programada había supuesto, además, la identificación y el desarrollo de destrezas hasta la fecha desconocidas para ellos mismos, ampliando de esta manera las llamadas destrezas de autodescubrimiento que permiten mejorar la autoestima, incitando con ello el desarrollo de la narrativa y el uso del lenguaje de base creativa.

\section{Discusión de resultados}

Los resultados de esta experiencia, como soporte para una práctica basada en la evidencia, verifican la mejora de los rendimientos académicos y la adquisición de competencias investigadoras por medio el aprendizaje reflexivamente crítico, utilizando la metodología Blended Learning mediante la instrumentalización de técnicas colabo- rativas y de aprendizaje significativo. Poder relacionar teoría y práctica del Trabajo Social, a través de acciones participativas, permite elaborar proyectos de investigación social que pueden ser compartidos virtualmente. En este sentido, coincidimos con Elliott (2010) cuando plantea que esta experiencia nos ha aventurado a pensar en el hecho de que la transformación del estudio de la enseñanza en estudio del aprendizaje identifica que la investigación-acción participante se lleva a cabo sobre el desarrollo de la práctica, y no sobre la teoría. Teniendo en cuenta esta afirmación, el cuestionamiento posterior ahondaría en el hecho de poder integrar la investigación en la práctica profesional de manera continuada, valorando su impacto tanto en la población a la que se atiende como en la evolución de la práctica cotidiana del Trabajo Social profesionalizado.

Si se focaliza en la práctica basada en la evidencia podemos trasladar los pasos que aportaba Mooney (2004) a nuestra realidad social, considerando los tres primeros momentos como esenciales para el diseño del proyecto de investigación social, y el último como esencial de nuestra práctica. En este sentido se propone un proceso basado en la evidencia que parta del proyecto de investigación social y termine en el proyecto de intervención, pero que a su ver retroalimente futuras investigaciones y asiente sus bases sobre cimientos científicos. 


\begin{abstract}
Proyecto de investigación social
Formulación del objeto de estudio: Basada en las necesidades del usuario de servicios sociales, este puede ser el individuo, el grupo o familia y/o la comunidad.

Investigación de la documentación en referencia a esta problemática, para ello es esencial la búsqueda en revistas científicas vinculadas con nuestra disciplina, proyectos de intervención en relación al objeto de estudio así como otro tipo de documentos, tal vez inéditos, pero accesibles (memorias, proyectos previos realizados, informes, etc.).

Valoración reflexiva y crítica del material localizado con el fin de adecuar esta documentación al objeto de estudio.

Ejecución del proyecto de investigación.

Evaluación de resultados del proyecto de investigación y realización de memoria.

\section{Proyecto de intervención social}

Diseño del proyecto de intervención.

Aplicación de la evidencia científica al objeto de estudio por medio de la experiencia y pericia profesional y coherente con el código deontológico de la profesión.

Evaluación de la intervención mediante ítems cuantificables.

Realización de informe de la intervención que servirá como base teórica para la futura práctica basada en la evidencia.
\end{abstract}

Cuadro 2. Proceso de intervención basada en la evidencia.

Nuestra disciplina, el Trabajo Social, ha sido identificada siempre con una práctica en contacto con la población y como una profesión mediadora entre las necesidades y los recursos disponibles (Sancho, 2005). En este sentido, los resultados derivados de la experiencia nos llaman poderosamente la atención ya que las competencias metodológicas de investigación (conocer y utilizar técnicas cuantitativas, cualitativas, de diseño, identificar bases de datos, búsquedas bibliográficas, etc.) son el apartado donde los alumnos mostraron más seguridad, pudiendo asumir este hecho una doble lectura: por un lado, el nuevo alumnado de Trabajo Social se ha empoderado de estrategias para la investigación (Barbero, 2006) con la intención de ampliar sus garantías diagnósticas y fortaleciendo la gestión efectiva de las dificultades sociales; pero por el otro lado, esa proximidad a la realidad introspectiva ¿no le alejará de su objeto de acción e intervención?, ¿le posicionará en un nivel poco actualizado, alejado de los problemas sociales con los que ha de interactuar?

Consideramos, como hemos visualizado en el Cuadro 2, que ambas partes, investigación e intervención, son absolutamente complementarias; pero será muy importante no perder de vista ni olvidar el contacto con lo cotidiano, porque en este caso habremos posicionado al Trabajo Social en un lugar de difícil retorno y alejado de lo que es nuestra disciplina, inmersa en la realidad social.

Un elemento esencial de esta experiencia es la creación del nexo entre el practicum que realiza el alumnado y la vida académica que, como consecuencia, nos permite prestar atención a la explicación de los fenómenos sociales (Beltrán, 1990; Hacking, 2001), permitiendo al alumnado que, a través de metodologías explicativas, sea capaz de analizar su entorno de intervención de modo colectivo, donde el plano individual forma parte de un tronco común que requiere el consenso para la acción. Es el punto concéntrico para la metodología Blended Learning de base colaborativa, donde se aceptan todas las propuestas, mediante una plataforma global y visible a todos, para discutirlas colectivamente y alcanzar consensos capaces de aunar todas las sensibilidades. Adaptando los parámetros que plantea Novoa (2009) para la educación, entendemos que esta dinámica es la esencia del actual Trabajo Social ya que, para alcanzar la autonomía de los individuos, es necesario una construcción colectiva de la realidad, no solamente capaz de partir de percepciones colectivas, sino también de la participación activa y dinámica de los sujetos, que en sintonía con Martínez, Casado e 
Ibarra (2012) trate de «compartir roles en la investigación, de tal forma que, todos/as nos asumimos como personas implicadas en el proceso y todos/as somos capaces de producir conocimientos válidos (y por supuesto, situados) sobre la realidad Investigada» (p. 30). En definitiva, esta metodología flexible, aplicada en el aula, tiene una validez absolutamente actualizada a la práctica cotidiana del Trabajo Social, en tanto que pone a su servicio la información y la participación con la intención final de responder a los patrones diagnósticos. Además de ello, pone en cuestión su propia adaptabilidad a la práctica profesional como estrategia para la excelencia, y actualiza el clásico concepto de diagnóstico social de Mary Richmond.

\section{Conclusiones}

A lo largo de esta experiencia se han demostrado, con la metodología novedosa, Blended Learning, las aportaciones que se pueden realizar al proyecto de investigación social como cimiento para la práctica basada en la evidencia en el Trabajo Social. En concreto, la intervención en esta disciplina requiere un diagnóstico reflexivo que debe emanar de la investigación científica y, en consecuencia, fortalecer las competencias en la materia empodera a los futuros profesionales y dota a nuestra disciplina de un mayor status en el ámbito académico. Vincular la teoría y la práctica a la creación de proyectos de investigación social que partan de las prácticas externas que realizan, aúna las vivencias inmediatas del alumnado, inmerso en la esencia definitoria de la profesión que es la intervención, con los elementos docentes e investigadores que aportan base científica a nuestra disciplina.
Este tipo de enseñanza es eminentemente coherente con nuestra profesión al centrar el aprendizaje en el estudiante, convirtiéndole en el intérprete principal de la enseñanza. Esto supone «un aprender aprendiendo», porque en la intervención en Trabajo Social serán los usuarios los protagonistas de su propia acción y, en consecuencia, empoderar a los alumnos en caminos emancipadores será sembrar intervenciones no anquilosadas en el tiempo.

Hablar de calidad de la educación hace referencia a la efectividad de la enseñanza-aprendizaje con la adaptación contextual a los escenarios, utilizando a nuestro favor elementos nuevos, como el aprendizaje a través de entornos virtuales, o más tradicionales, como el trabajo colaborativo, la clase presencial o la tutoría individual, que facilitan la adquisición de conocimientos y que mejoran el rendimiento académico de manera permanente. Asimismo los resultados de la realización del taller nos demuestran la capacidad para poder adaptar estas metodologías a la práctica cotidiana del Trabajo Social, como una herramienta adicional capaz de incentivar las respuestas colectivas ante el abordaje de las problemáticas sociales, nos abre el camino a nuevas formas de relación en los equipos interdisciplinares.

Como conclusión, hemos de subrayar que el proyecto de investigación social representa la raíz en la que crecen los diagnósticos fiables de la realidad y, en consecuencia, acrecientan buenas intervenciones tales como serán las prácticas basadas en la evidencia. Esto aporta una base sólida de conocimientos y, como resultado, no sólo un mayor status para la profesión sino una mejora de la práctica y, por ende, del bienestar de las personas con las que intervenimos, que al fin y al cabo es de lo que se trata.

\section{Referencias bibliográficas}

Aguado, D. y Arranz, V. (2005). Desarrollo de competencias mediante blended learning: un análisis descriptivo. Pixel-Bit, Revista de medios y educación, 26, 79-88. Disponible en: http://www. rieoei.org/deloslectores/1118Arranz.pdf (Consultado el 19 de abril de 2013).

Aillo, M. (2004). El Blended Learning como práctica transformadora. Revista Píxel-Bit, 23, 21-26. Disponible en: http://www.sav.us.es/pixelbit/pixelbit/articulos/n23art/art2302.htm (Consultado el 16 de diciembre de 2012).

Aronson, E. y Patnoe, S. (2011). The Jigsaw Classroom. Building Cooperation in the Classroom. (3 edición). Estados Unidos: Longman.

Ausubel, D. P. (2000). The adquisition and retention of knowledge: a cognitive view. Dordrecht, Boston: Kluwer Academic Publishers.

Barbero, J.M. (2006). La autogestión del Trabajo Social y la perspectiva del investigador. Cuadernos de Trabajo Social, 19, 43-54. 
Beltrán, M. (1990). Cinco vías de acceso a la realidad social. En M. García Ferrando, J. Ibáñez y F. Alvira (1990). El análisis de la realidad social: Métodos y técnicas de investigación. Madrid: Alianza Editorial.

Chickering, A.W. y Gamson, Z.F. (1987). Seven principles for good practice in undergraduate education. The Wingspread Journal, 9 (2), 1-15.

Coll, C., Maurí, T. y Honrubia, J. (2006). Análisis y resolución de casos-problema mediante el aprendizaje colaborativo. Revista de Universidad y Sociedad del Conocimiento, 3(2), 29-41.

Domingo, J. (2008). El aprendizaje cooperativo. Cuadenos de Trabajo Social, 21, 231-246.

Cabero, J. y Llorente, M.C. (2005). Las plataformas virtuales en el ámbito de la teleformación. Revista electrónica Alternativas de educación y comunicación,. 1-24.

Elliott, J. (2010). El «estudio de la enseñanza y del aprendizaje»: una forma globalizadora de investigación del profesorado. Revista interuniversitaria de formación del profesorado, 68, 223-242.

Freire, P. (1979). Educación y acción cultural. Bilbao: Zero.

Garaigordobil, M. (2007). Los juegos cooperativos: una nueva forma de juego para construir una sociedad más justa y solidaria. Madrid: Crítica.

Garaigordóbil, M. (2012). Cooperative conflict-solving during adolescence: relations with cognitive-behavioral variables and predictors. Infancia y Aprendizaje, 35(2), 151-165.

Gil, F., Rico, R. y Sánchez-Manzanares, M. (2008). Eficacia de equipos de trabajo. Papeles del Psicólogo, 29, 25-31.

Hacking, I. (2001). ¿La construcción social de qué? Barcelona: Paidós.

Johnson, D.W. y Johnson, R. (1990). Cooperation and competition. theory and research. Hillsdale, NuevaYork: Addison-Wesley.

Johnson, D., Johnson, R. y Holubec, E. (1999). Aprendizaje cooperativo en el aula. Buenos Aires: Paidós.

Pastor, E. (2011). Capital social, inteligencia cooperativa y diálogo significante en el ámbito local: dimensiones de análisis e intervención para intensificar la participación. Revista de Investigaciones Políticas y Sociológicas, 10 (2), 9-27.

Martínez, Z., Casado, B. e Ibarra, P. (2012). Movimientos sociales y procesos emancipadores. Cuadernos de Trabajo Hegoa, 57, 3-40.

Mooney, G.H (2004). Evidence Based Medicine: In Its Place. Londres: Routledge

Morago, P. (2009). La práctica basada en la evidencia en los cursos de titulación de Trabajo Social. Cuadernos de Trabajo Social. 22, 41-59

Moreira, M A. (2000). Aprendizaje significativo: teoría y práctica. Madrid: VISOR.

Novoa, A. (2009). Para una formación de profesores construida dentro de la profesión. Revista de educación. 350, 203-218

Salinas, J. (2003): Comunidades Virtuales y Aprendizaje digital. Ponencia. EDUTEC'03. VI Congreso Internacional de Tecnologia Educativa y Nuevas Tecnologías aplicadas a la Educación: Gestión de las Tecnologías de la Información y la Comunicación en los diferentes ámbitos educativos (Universidad Central de Venezuela, Caracas, 24-27 noviembre). Recuperado de: http://gte.uib.es/pages/castella/comunidades_virtuales.pdf

Sancho, J. (2005). Exclusión social en la sociedad de la información. Revista de Servicios Sociales y Política Social, 55, 55-67.

Silva, R. (2011). La enseñanza de la fisica mediante un aprendizaje significativo y cooperativo en Blended Learning (Tesis doctoral. Universidad de Burgos, Burgos).

Sackett, D.L., Straus, S.E., Richardson, W.S., Rosenberg, W. y Haynes, R.B. (2000) Evidence-Based Medicine: How to Practice and Teach EBM, (2 ${ }^{\mathrm{a}}$ ed.). Nueva York: Churchill Livingstone.

Slanvin, R.E. y Cooper, R. (1999). Improving intergroup relations: Lessons learned from cooperative learning programs. Journal of Social Issues, 55, 647-663.

Susman, E.B. (1998). Cooperative learning: A review of factors that increase the effectiveness of cooperative computer-based instruction. Journal of Educational Computing Research, 18. 